\title{
Recent progress in fluctuation theorems and free energy recovery
}

\author{
A. Alemany*, M. Ribezzi* and F. Ritort ${ }^{*}$, \\ *Small Biosystems Lab, Departament de Fisica Fonamental, Facultat de Fisica, Universitat de \\ Barcelona, Diagonal 647, 08028 Barcelona, Spain \\ ${ }^{\dagger}$ CIBER-BBN, Networking Center of Bioengineering, Biomaterials and Nanomedicine, ISCIII, \\ Madrid, Spain
}

\begin{abstract}
In this note we review recent progress about fluctuation relations and their applicability to free energy recovery in single molecule experiments. We underline the importance of the operational definition for the mechanical work and the non-invariance of fluctuation relations under Galilean transformations, both aspects currently amenable to experimental test. Finally we describe a generalization of the Crooks fluctuation relation useful to recover free energies of partially equilibrated states and thermodynamic branches.
\end{abstract}

Keywords: Nonequilibrium systems, fluctuation theorems,single-molecule experiments, optical tweezers

PACS: $87.14 . \mathrm{Ee}, 82.20 . \mathrm{Db}, 87.15 . \mathrm{Cc}$

\section{NONEQUILIBRIUM SMALL SYSTEMS}

In 1944 Erwin Schrödinger published the classic monography What is life? where he pointed out the importance of physical and chemistry laws to understand living systems [1]. The notion that genetic information should be encoded in an "aperiodic crystal" seeded the subsequent discovery of the double helix structure of DNA. Chapter 7 of Schrödinger's monography contains an interesting discussion about the similarities and differences between a clockwork motion and the functioning of an organism. According to Schrödinger the regular motion of a clock must be secured by a weak spring. Yet, whatever the weakness of the spring is, it will produce frictional effects that do compensate for the external driving of the clock (e.g. the battery) in order to secure its regular motion. Being friction a statistical phenomenon he concludes that the regular motion of the clock cannot be understood without statistical mechanics. Then he further states: For it must not believed that the driving mechanism really does away with the statistical nature of the process. The true physical picture includes the possibility that even a regularly going clock should all at once invert its motion and, working backward, rewind its own spring -at the expense of the heat of the environment. The event is just 'still a little less likely' than a 'Brownian fit' of a clock without driving mechanism.

Recent advances in microfabrication techniques, detection systems and instrumentation have made possible the measurement of such "inverted motions" referred by Schrödinger. The controlled manipulation and detection of very small objects makes possible to exert and measure tiny forces applied on them and follow their trajectories in space-time with resolution of piconewtons, nanometers and microseconds respectively. 
According to the equipartition law, systems with a low number of degrees of freedom embedded in a thermal environment exhibit energy fluctuations that are a few times $k_{\mathrm{B}} T$ ( $k_{\mathrm{B}}$ being the Boltzmann constant and $T$ the environmental temperature). Techniques such as atomic force microscopy (AFM), optical tweezers (OT) and magnetic tweezers (MT) allow for the controlled manipulation of individual molecules such as nucleic acid structures and proteins [2], the measurement of very small energies (within the $k_{\mathrm{B}} T$ scale) [23] and the observation of "inverted motion" in translocating enzymes [3]. These developments during the past years have been accompanied by a concomitant progress of theoretical results in the domain of nonequilibrium physics [4]. This contribution reviews some of the most basic concepts around fluctuation theorems and their experimental verification.

\subsection{Control parameters, configurational variables and the definition of work}

In small systems it is crucial to make a distinction between controlled parameters and non-controlled or fluctuating variables. Controlled parameters are those macroscopic variables that are imposed on the system by the external sources (e.g. the thermal environment) and do not fluctuate with time. In contrast, non-controlled variables are microscopic quantities describing the internal configuration of the system and do fluctuate in time because they are subject to Brownian forces. Let us consider a typical single molecule experiment where a protein is pulled by an AFM. In this case the control parameter is given by the position of the cantilever that determines the degree of stretching and the average tension applied to the ends of the protein. Also the temperature and the pressure inside the fluidic chamber are controlled parameters. However, the height of the tip respect to the substrate or the force acting on the protein are fluctuating variables describing the molecular extension of the protein tethered between tip and substrate. Also the position of each of the residues along the polypeptide chain are fluctuating variables. Both molecular extension or force and the residues positions define different types of configurational variables. However only the former are subject to experimental measurement and therefore we will restrict our discussion throughout this paper to such kind of experimentally accessible configurational variables. Figure 1 illustrates other examples of control parameters and configurational variables. In what follows we will denote by $\lambda$ the set of controlled (i.e. non-fluctuating) parameters and $x$ the set of configurational (i.e. fluctuating) variables. The definition of what are controlled parameters or configurational variables is broad. For example, a force can be a configurational variable and a molecular extension can be a controlled parameter, or vice versa, depending on the experimental setup (see Figure 1, right example).

The energy of a system acted by external sources can be generally described by a Hamiltonian or energy function, $U(x, \lambda)$. The net variation of $U$ is given by the conservation law,

$$
d U=\left(\frac{\partial U}{\partial x}\right) d x+\left(\frac{\partial U}{\partial \lambda}\right) d \lambda=d Q+d W
$$




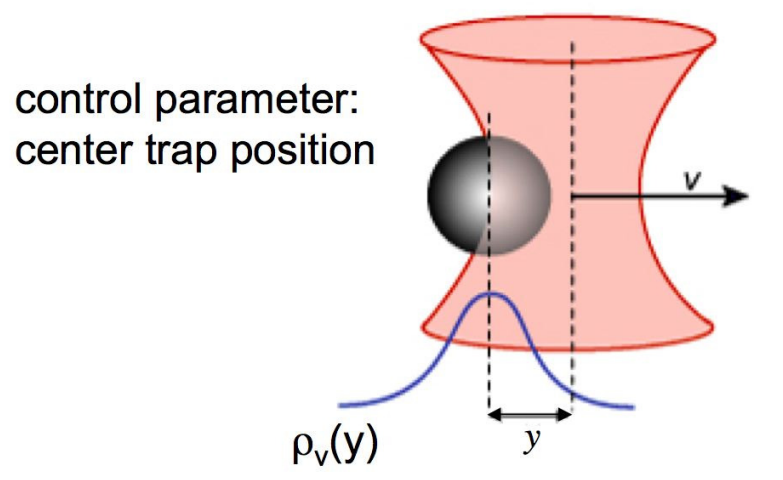

\section{control parameter: external force}
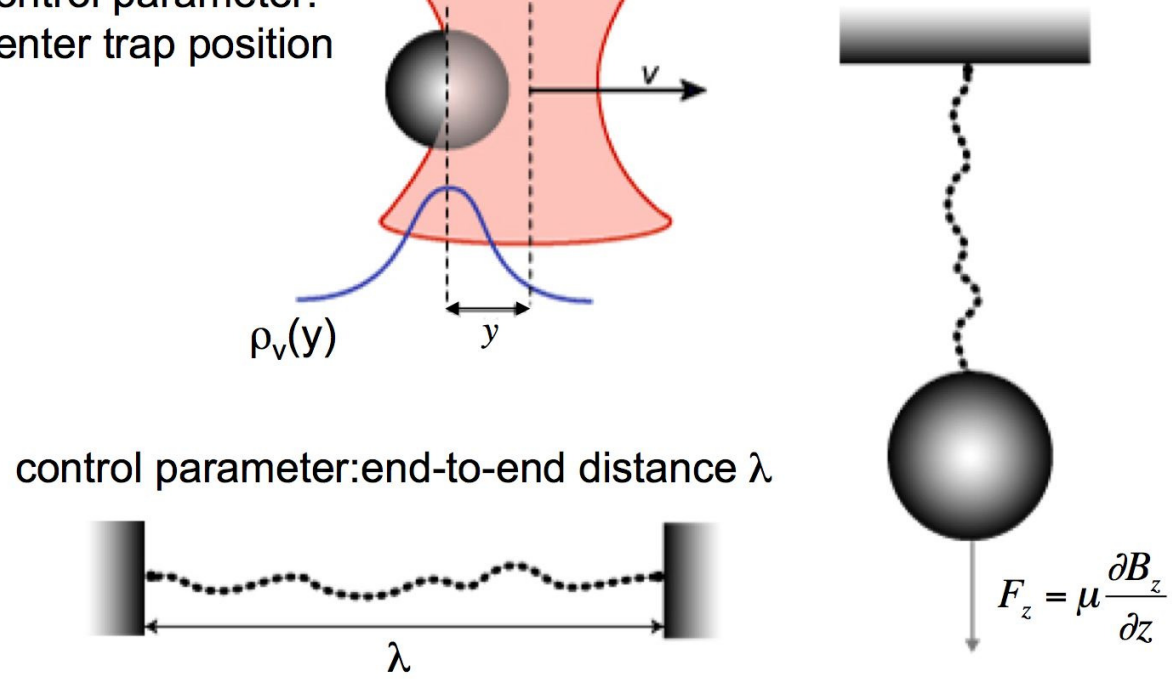

FIGURE 1. Control parameter and configurational variables. Different experimental setups corresponding to different types of control parameters (denoted as $\lambda$ ) or configurational variables (denoted as $x$ ). (Top left) A micron-sized bead dragged through water. $\lambda$ could be the center of the trap measured in the lab (i.e. fixed to the water) frame whereas $x$ is the displacement of the bead, indicated as $y$, respect to the center of the trap. (Bottom left) A polymer tethered between two surfaces. $\lambda$ is the distance between the surfaces and $x$ the force acting on the polymer. (Right) A polymer stretched with magnetic tweezers. $\lambda$ is the force acting on the magnetic bead and $x$ is the molecular extension of the tether. Figure taken from [3].

where $d Q, d W$ stand for the infinitesimal heat and work transferred to the system. The previous mathematical relation has simple physical interpretation. Heat accounts for the energy transferred to the system when the configurational variables change at fixed value of the control parameter. Work is the energy delivered to the system by the external sources upon changing the control parameter for a given configuration. The total work performed by the sources on the system when the control parameter is varied from $\lambda_{i}$ to $\lambda_{f}$ is given by,

$$
W=\int_{\lambda_{i}}^{\lambda_{f}} d W=\int_{\lambda_{i}}^{\lambda_{f}}\left(\frac{\partial U}{\partial \lambda}\right) d \lambda=\int_{\lambda_{i}}^{\lambda_{f}} F(x, \lambda) d \lambda
$$

where $F(x, \lambda)$ is a generalized force defined as,

$$
F(x, \lambda)=\left(\frac{\partial U}{\partial \lambda}\right)
$$

It is important to stress that the generalized force is not necessarily equal to the mechanical force acting on the system. In other words, $F(x, \lambda)$ is a configurational dependent variable conjugated to the control parameter $\lambda$ and has dimensions of [energy]/[ $\lambda]$ 
which are not necessarily Newtons. In the example shown in the right of Figure 1 the control parameter is the magnetic force $\lambda \equiv f$ and the configurational variable $x$ is the molecular extension of the polymer. The total Hamiltonian of the system is then given by $U(x, f)=U_{0}(x)-f x$ where $U_{0}(x)$ is the energy of the system at $\lambda=f=0$. In other words, the external force $f$ shifts all energy levels (defined by $x$ ) of the original system by the amount $-f x$. The generalized force is then given by $F(x, f)=-x$ (i.e. it has the dimensions of a length) and $d W=-x d f$. The fact that $d W$ is equal to $-x d f$ and not equal to $f d x$ has generated some controversy [5]. Below we show how this distinction is already important for the simplest case of a bead in the optical trap. In Section 2.1 we also show how the physically sound definition of mechanical work is amenable to experimental test.

\subsection{A classical experiment: the bead in the optical trap}

In 2002 Dennis Evans and coworkers in Camberra (Australia) performed the first experiment where the "inverted motions" could be observed [6]. The experiment is shown in Figure 2a. A micron-sized spherical bead made of silica or polystyrene is immersed in water inside a fluidic chamber and captured in an optical trap of infrared light generated by a high numerical aperture objective. Initially the trap is at a rest position and the bead is in thermal equilibrium and fluctuating around the center trap position. Suddenly the trap is set into motion at a constant speed $v$ and the bead is dragged through the water. After a transient time $\tau=\gamma / k$ the bead reaches a steady state where the Stokes frictional force is counter balanced by the optical trapping force. The average bead position lags behind the center of the trap by a distance $\bar{y}=\gamma v / k$ where $\gamma=6 \pi \eta R$ is the friction coefficient ( $\eta$ is the water viscosity and $R$ is the bead radius) and $k$ is the stiffness of the trap. In the laboratory frame (see Figure 2a) the bead and trap center have coordinates $x(t)$ and $x^{*}(t)=v t$ (we take $x=x^{*}=0$ at $t=0$ when the trap is set in motion). The distance between the center of the trap and the bead is $y=x^{*}-x$ and the restoring force acting on the bead is given by $F(y)=k y$. In this example the control parameter is given by the trap center $\lambda=x^{*}$ whereas $x$ is the configurational variable. The trapping energy of the bead is given by $U(x, \lambda)=(1 / 2) k(x-\lambda)^{2}$ and the generalized force $F=k(\lambda-x)=k y$ (cf. Eq.(3)). The work exerted by the trap on the bead is then equal to $W=\int_{0}^{t} F(y) d x^{*}=v k \int_{0}^{t} y(s) d s$. The first remarkable fact in this expression is that the work $W$ is neither equal to $W^{\prime}=\int_{0}^{t} F(y) d x$ nor $W^{\prime \prime}=\int_{0}^{t} F(y) d y$. These three quantities have different physical meaning. In fact, by exactly integrating the force, $W^{\prime \prime}$ becomes equal to the energy difference between the initial and final configurations. Whereas $W^{\prime}$ is equal to minus the heat, $-Q$. Since $d y=d x^{*}-d x$ what we are now facing is again the mathematical statement of energy conservation. Note also that the work definition is non-invariant under Galilean transformations. In fact, the work definition requires that $x$, as measured in the lab frame, is the proper configurational variable. If we choose $y$ rather than $x$ ( $y$ is now measured in the trap-moving frame) then $U(y, \lambda)=(1 / 2) k y^{2}$ is independent of $\lambda$ and the work would be identically zero which makes no sense. 


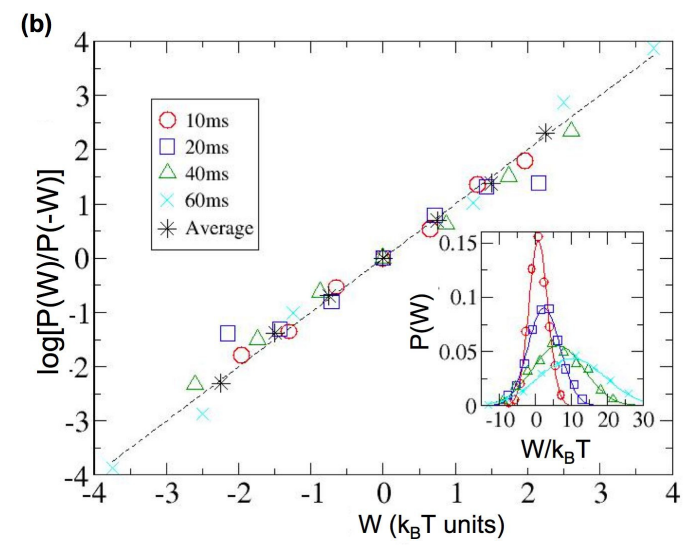

FIGURE 2. The bead in the optical trap dragged through water. (a) Variables defining the experiment. (b) Work distribution measurements corresponding to different elapsed times. The mean work $\bar{W}$ and variance $\sigma_{W}^{2}$ increase with time (Inset). The main panel shows the experimental test of the fluctuation relation Eq.(7) for all experimental data put together.

By repeating the moving trap experiment an infinite number of times a work distribution will be produced. The shape of the work distribution must be Gaussian because the stochastic variable $y$ follows an Ornstein-Uhlenbeck process that can be described by a linear Langevin equation. Consequently, only the first and second cumulants of the work distribution are non-zero. Let us note in passing that the Gaussian property is not fulfilled by the heat and the energy difference, which are known to exhibit exponential tails $[8,9]$. The mean work $\bar{W}$ and variance $\sigma_{W}^{2}=\overline{W^{2}}-\bar{W}^{2}$ can be easily worked out in the asymptotic regime for times $t$ that are longer than the relaxation time of the bead, $t>>/ k$. In this limit,

$$
\begin{array}{r}
W=v k \int_{0}^{t} \overline{y(s)} d s \rightarrow v k \bar{y} t=\gamma v^{2} t \\
\sigma_{W}^{2}=\overline{W^{2}}-\bar{W}^{2}=v^{2} k^{2} \int_{0}^{t} \int_{0}^{t} \overline{y\left(s_{1}\right) y\left(s_{2}\right)} d s_{1} d s_{2} \rightarrow 2 v^{2} k^{2} t \int_{0}^{t} \overline{y(0) y(s)} d s= \\
=2 v^{2} k^{2} t \overline{y^{2}} \tau=2 v^{2} k^{2} t \frac{k_{\mathrm{B}} T}{k} \frac{\gamma}{k}=2 k_{\mathrm{B}} T \gamma v^{2} t
\end{array}
$$

where $\ldots$ stands for an average over trajectories. In deriving Eq.(5) we used timetranslational invariance in the steady state and the result $\overline{y(0) y(s)}=\overline{y(0)^{2}} \exp (-s / \tau)$ in the steady state ( $\tau=\gamma / k$ being the bead relaxation time) with $\overline{y(0)^{2}}=k_{\mathrm{B}} T / k$ due to the equipartition law. The work probability distribution is finally given by,

$$
P(W)=\frac{1}{\sqrt{2 \pi \sigma_{W}^{2}}} \exp \left(-\frac{(W-\bar{W})^{2}}{2 \sigma_{W}^{2}}\right) .
$$

These relations teach us various things:

1. Second law. The mean work $\bar{W}$ is always positive (second law) and only vanishes at all times for $v \rightarrow 0$, i.e. when the trap is moved in a quasistatic way. 
2. Observation of "Inverted motions". Although $\bar{W}>0$ there are always trajectories for which $W<0$. These are the "inverted motions" refereed to by Schrödinger and recently renamed as "transient violations of the second law". Along these "inverted motion" trajectories the bead extracts energy from heat fluctuations to overcome the frictional forces and to move ahead of the center of the trap.

3. "Inverted motions" as rare events. Both the mean work and the standard deviation of the work increase with time and trap speed. However the standard deviation $\sigma_{W}$ increases as $\sqrt{t}$ whereas $\bar{W}$ increases faster (linearly with $t$ ). Therefore, although it is possible to find trajectories where $W<0$, these are rare events because their relative fraction decreases exponentially fast with time. $W<0$ trajectories become more probable (i.e. less rare) at short times. In the limit $t \rightarrow 0$ they reach the $50 \%$ of all events.

4. Fluctuation relation. The work probability density function shown in Eq.(6) satisfies a fluctuation relation. From Eqs. $(4,5)$ we find $\sigma_{W}^{2}=2 k_{\mathrm{B}} T \bar{W}$. It is straightforward to check that the following relation holds,

$$
\frac{P(W)}{P(-W)}=\exp \left(\frac{W}{k_{\mathrm{B}} T}\right) \quad .
$$

Eq.(7) receives the name of a fluctuation relation because it is an exact mathematical relation describing arbitrarily large work fluctuations. Eq.(7) was derived from Eq.(6) which was obtained in the limit of long enough times. More elaborate calculations show that this relation is exact for arbitrary times [7, 8].

In Figure $2 \mathrm{~b}$ we show an experimental test of these results. The fluctuation relation in Eq.(7) corresponds to a special case of what is known as transient fluctuation theorem (TFT) [10]. The system is initially in equilibrium and transiently driven out of equilibrium by external forces. The generalization of such relation to include arbitrary nonequilibrium transient states gives the fluctuation relation by Crooks described in the next section.

\section{THE CROOKS FLUCTUATION RELATION AND FREE ENERGY RECOVERY.}

Let us consider a generic system in thermal equilibrium that is transiently driven out of equilibrium during the time interval $\left[0, t_{f}\right]$ by varying $\lambda$ according to a protocol $\lambda(t)$ from an initial value $\lambda(0)=\lambda_{i}$ to a final value $\lambda\left(t_{f}\right)=\lambda_{f}$. We refer to this as the forward (F) process. By repeating this process an infinite number of times we generate the work distribution $P_{F}(W)$. Let us consider now the reverse process where the system starts in equilibrium at $\lambda_{f}$ and $\lambda$ is varied according to the time reversal protocol, $\lambda\left(t_{f}-t\right)$, until reaching the final value $\lambda_{i}$ (see Figure 3 ). The reverse (R) process can be repeated an infinite number of times to produce the work distribution $P_{R}(W)$. The Crooks fluctuation relation $(\mathrm{CFR})$ reads [11],

$$
\frac{P_{F}(W)}{P_{R}(-W)}=\exp \left(\frac{W-\Delta G}{k_{\mathrm{B}} T}\right)
$$


(a)

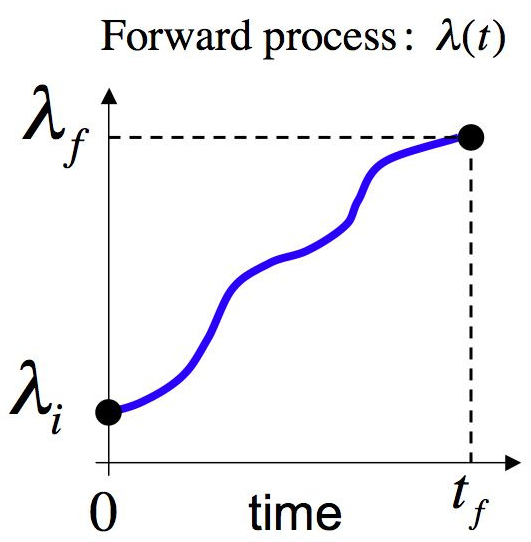

(b)

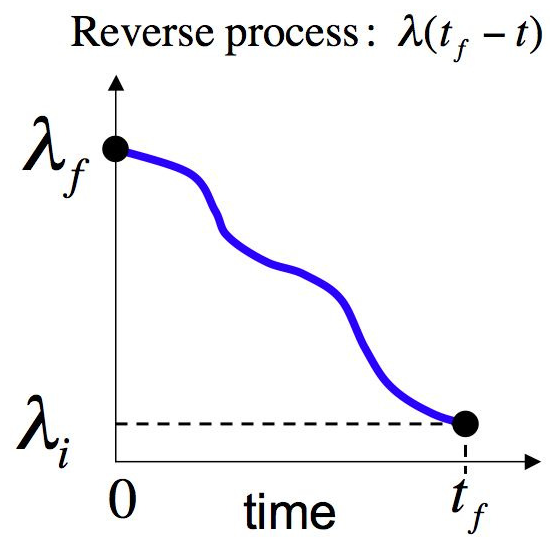

FIGURE 3. Forward and reverse paths. (a) An arbitrary forward protocol. The system starts in equilibrium at $\lambda_{i}$ and is transiently driven out of equilibrium until $\lambda_{f}$. At $\lambda_{f}$ the system may be or not in equilibrium. (b) The reverse protocol of (a). The system starts in equilibrium at $\lambda_{f}$ and is transiently driven out of equilibrium until $\lambda_{i}$. At $\lambda_{i}$ the system may be or not in equilibrium.

where $\Delta G=G\left(\lambda_{f}\right)-G\left(\lambda_{i}\right)$ is equal to the free energy difference between the equilibrium states at $\lambda_{f}$ and $\lambda_{i}$. Eq.(7) is a particular case of the CFR where $P_{F}(W)=P_{R}(W)$ (the trapping potential is symmetric $V(y)=V(-y)$ ) and $\Delta G=0$ (the free energy of the bead in the trap does not depend on the position of the trap, $\left.x^{*}\right)$. A particular result of the CFR is the well-known Jarzynski equality [12], $\overline{\exp \left(-W / k_{\mathrm{B}} T\right)}=\exp \left(-\Delta G / k_{\mathrm{B}} T\right)$, that has been used for free energy recovery $[13,14]$ by inverting the mathematical identity, $\Delta G=-k_{\mathrm{B}} T \log \left(\overline{\exp \left(-W / k_{\mathrm{B}} T\right)}\right)$. However this expression is strongly biased for a finite number of measurements $[15,16]$. Bidirectional methods that combine information from the forward and reverse processes and use the CFR have proven more predictive $[17,18,19]$. In particular the CFR immediately implies that $P_{F}(W)=P_{R}(-W)$ for $W=\Delta G$ showing that it is possible to measure $\Delta G$ in irreversible processes by measuring the forward and reverse irreversible work distributions and looking for the value of $W$ where they cross each other. The CFR was experimentally tested in 2005 in RNA pulling experiments with laser tweezers [20] showing this to be a reliable and useful methodology to extract free energy differences between states that could not be measured with bulk methods.

In Figure 4 we summarize recent results obtained in the Small Biosystems lab in Barcelona for the mechanical unfolding/refolding of DNA hairpins [21, 22] using a dualbeam miniaturized optical tweezers [23]. DNA hairpins are versatile structures formed by a stem of a few tens of base pairs that end in loop. They have some advantages as compared to RNA structures such as the easier synthesis and larger chemical stability. DNA hairpins can be easily synthesized and ligated to dsDNA handles to produce a construct ready to be pulled with the tweezers [24]. By chemically labeling the ends of the dsDNA handles it is possible to tether a DNA construct (formed by the DNA hairpin inserted between the two flanking handles) between two micron sized beads. One bead is immobilized in the tip of a pipette. The other bead is captured in the optical trap. The deflected light by the trapped bead provides a direct measurement of the force 
(a)

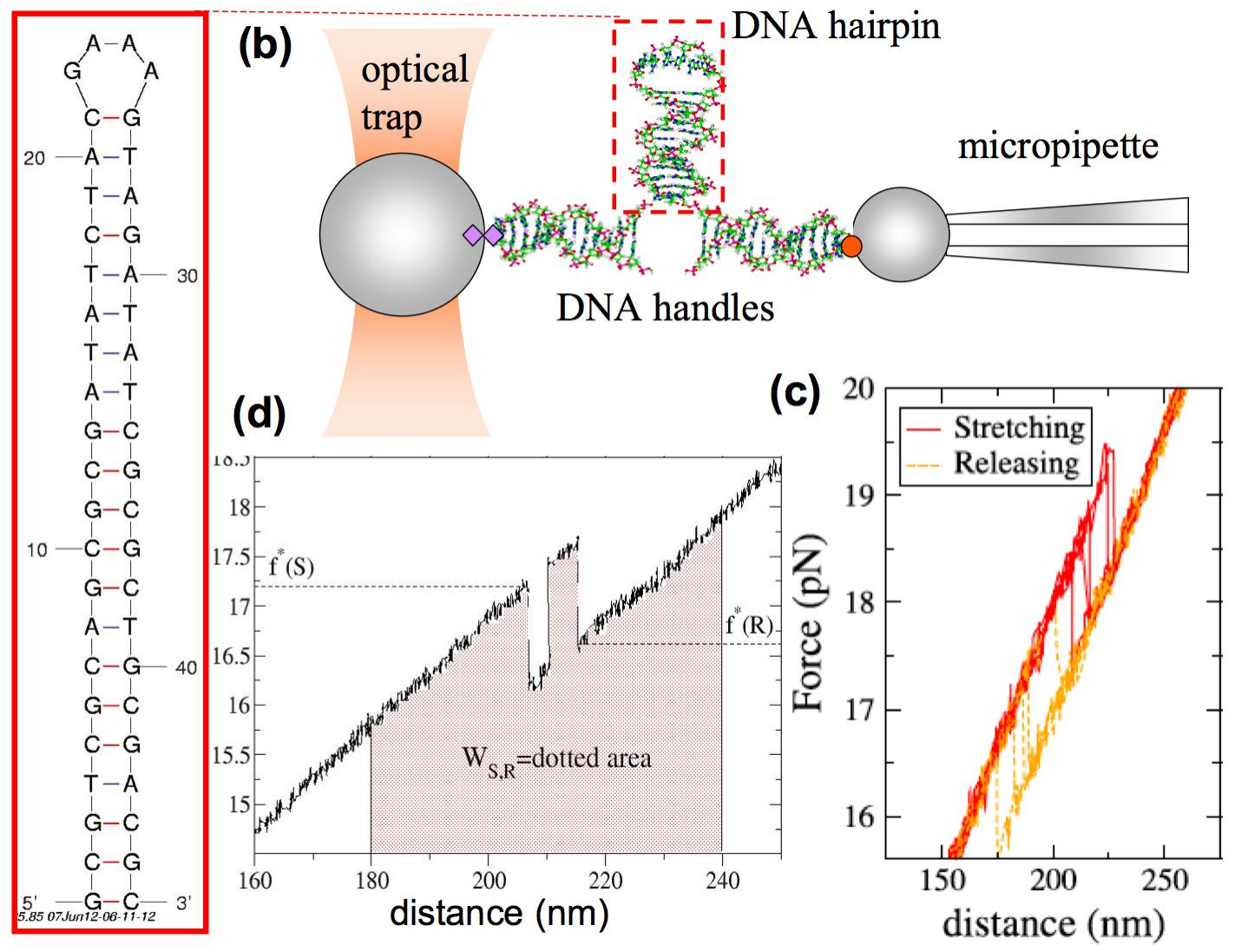

FIGURE 4. Mechanical folding/unfolding of DNA hairpins.(a) The sequence of a DNA hairpin with a 21 bp stem ending in a tetraloop. (b) Experimental setup. A molecular construct made of the hairpin shown in (a) flanked by two dsDNA handles (29bp each) is tethered between two micron-sized beads. In the experiments the trap is moved relative to the pipette at speeds ranging from 10 to $1000 \mathrm{~nm} / \mathrm{s}$. (c) Different force cycles recorded at $300 \mathrm{~nm} / \mathrm{s}$. The red curves indicate the stretching parts of the cycle whereas the orange curves indicate the releasing parts of the cycle. Note that the forces of unfolding and refolding are random due to the stochastic nature of the thermally activated unfolding/folding process. The marked hysteresis is a signature of an irreversible process. (d) Measurement of work for a single trajectory. It is given by the area below the force-distance curve integrated between two trap positions. Trap distances are relative. Note that there might be more than one unfolding or refolding event along each trajectory. $f^{*}(S)\left(f^{*}(R)\right)$ defines the first rupture force in the unfolding (refolding) process. Figure taken from [21].

applied on the molecule. By repeatedly steering the optical trap back and forth it is possible to unfold and refold the hairpin structure many times until the tether breaks. The unfolding of the hairpin is revealed by a sudden drop in the force due to the increase in molecular extension from the released single-stranded DNA of the hairpin. Such increase causes a retraction in the position of the bead in the trap and a force drop. Analogously, when the hairpin refolds a sudden increase in force is observed. One of the most successful constructs we have designed in our lab consists of DNA hairpins linked to two beads via extremely short (29bp) dsDNA handles [25]. These constructs are found to moderately increase the signal-to-noise ratio of the measurements allowing for precise work measurements. In a pulling experiment the force versus the relative trap-pipette 

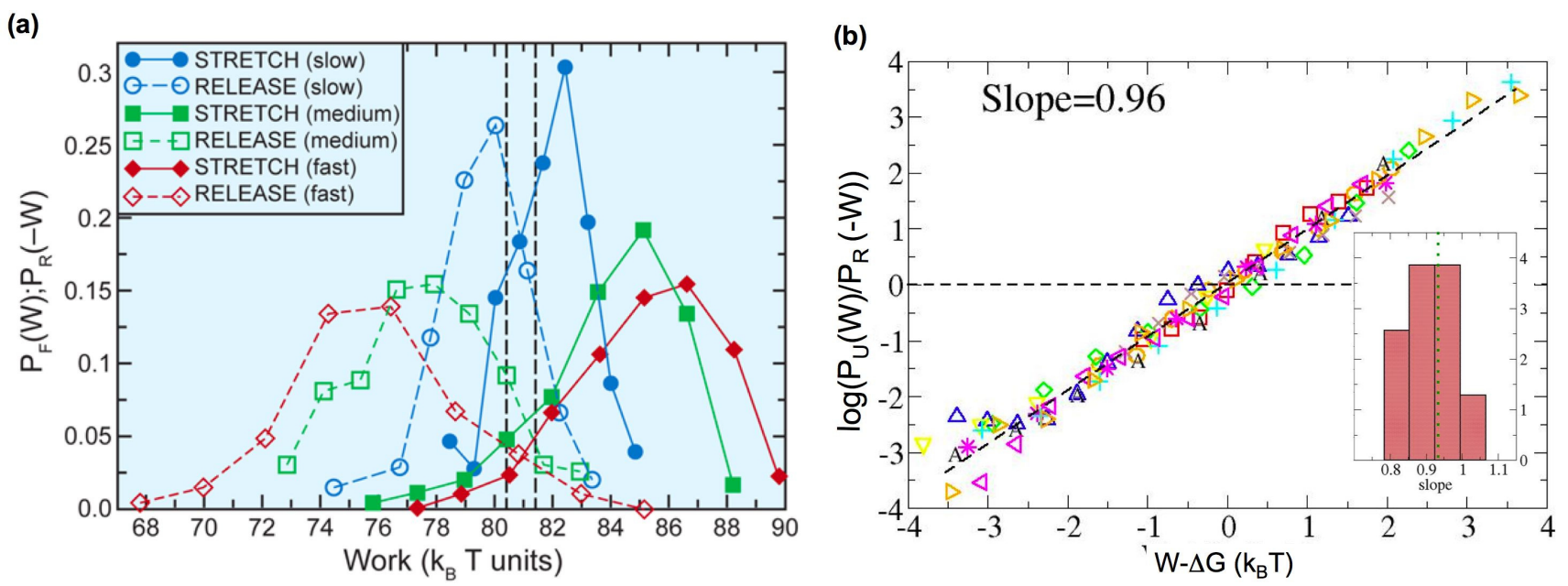

FIGURE 5. The Crooks fluctuation relation.(a) Work distributions for the hairpin shown in Figure 4 measured at three different loading rates: $50 \mathrm{~nm} / \mathrm{s}$ (blue), $100 \mathrm{~nm} / \mathrm{s}$ (green) and $300 \mathrm{~nm} / \mathrm{s}$ (red). Unfolding or forward (continuous lines) and refolding or reverse work distributions (dashed lines) cross each other at a a value $\simeq 81 k_{\mathrm{B}} T$ independent of the loading rate.(b) Experimental test of the CFR for 10 different molecules pulled at different speeds. The log of the ratio between the unfolding and refolding work distributions is equal to $(W-\Delta G)$ in $k_{\mathrm{B}} T$ units. The inset shows the distribution of slopes for the different molecules which are clustered around an average value of 0.96 . Figure taken from [21].

distance is recorded and the area below that curve provides a direct measurement of the work. Repeated measurements of the work make possible an experimental verification of the CFR (see figure 5).

\subsection{About the right definition of work: accumulated versus transferred work}

In a pulling experiment there are two possible representations of the pulling curves (Figure 6b). In one representation the force is plotted versus the relative trap-pipette distance $(\lambda)$, the so-called force-distance curve (hereafter referred as FDC). In the other representation the force is plotted versus the relative molecular extension $(x)$, the socalled force-extension curve (hereafter referred as FEC). In the optical tweezers setup $\lambda=x+y$ where $y$ is the distance between the bead and the center of the trap. The measured force is given by $F=k y$ where $k$ is the stiffness of the trap. The areas below the FDC and the FEC define two possible work quantities, $W=\int_{\lambda_{i}}^{\lambda_{f}} F d \lambda$ and $W^{\prime}=\int_{x_{i}}^{x_{f}} F d x$. From the relation $d \lambda=d x+d y$ we get,

$$
W=W^{\prime}+W_{b}=W^{\prime}+\frac{F_{f}^{2}-F_{i}^{2}}{2 k}
$$

where $F_{i}, F_{f}$ are the initial and final forces along a given trajectory. $W$ is often called the total accumulated work and contains the work exerted to displace the bead in the trap, $W_{b}$, and the work transferred to the molecular system, $W^{\prime}$ (therefore receiving the 
(a)

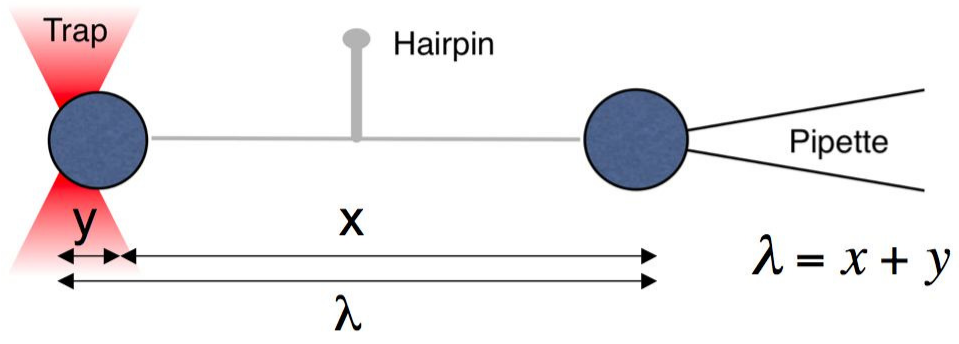

(b)
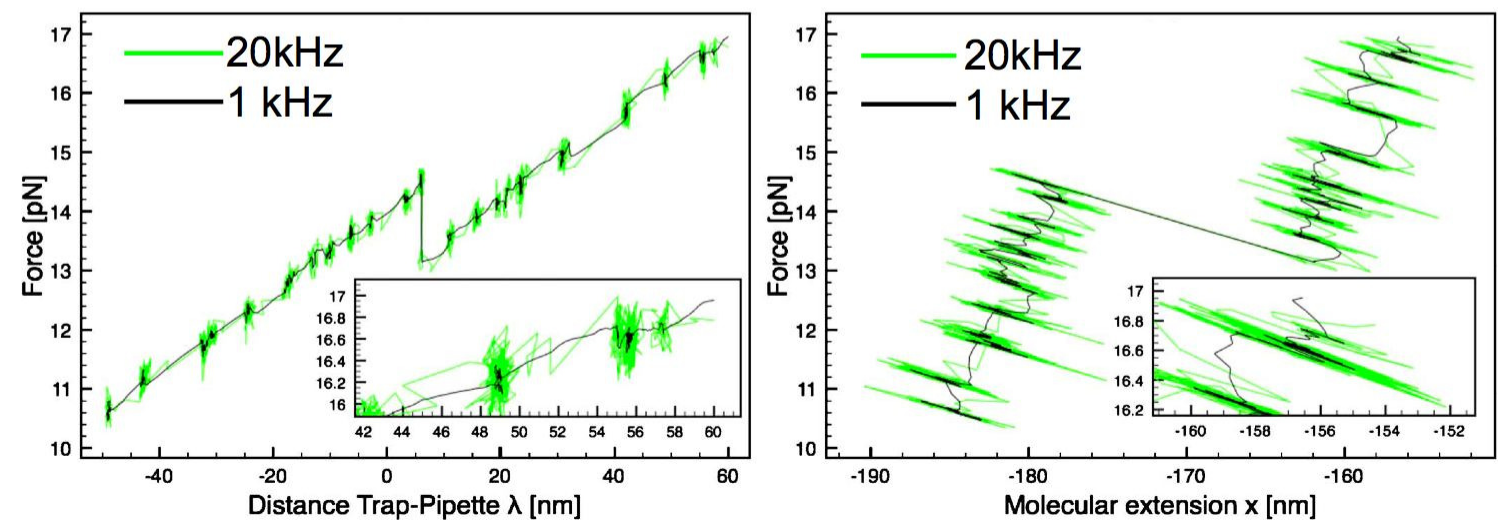

FIGURE 6. FDC versus FEC. (a) Experimental setup and different variables. (b) The FDC and FEC are defined as the curves obtained by plotting the force versus the trap position or the molecular extension respectively. Although force fluctuations in both types of curves show a dependence with the bandwidth of the measurement (black, $1 \mathrm{kHz}$; green $20 \mathrm{kHz}$ ) only in the FEC the measurement of the work is very sensitive to such fluctuations. Figure taken from [27].

name of transferred work) [26, 27]. The term $W_{b}$ appearing in Eq.(9) implies that $W, W^{\prime}$ cannot simultaneously satisfy the CFR. What is the right definition of the mechanical work? In other words, which work definition satisfies the CFR? The problem we are facing now is identical to the one we previously encountered in section 1.2 where we had to distinguish between work and heat. The answer to our question is straightforward if we correctly identify which are the control parameters and which are the configurational variables. In the lab frame defined by the pipette (or by the fluidic chamber to which the pipette is glued) the control parameter $\lambda$ is given by the relative trap-pipette distance, whereas the molecular extension $x$ stands for the configurational variable. Note that, due to the non-invariance property of the CFR under Galilean transformations, $y$ cannot be used as configurational variable because it is defined respect to the co-moving frame defined by the trap. The same problem was found in section 1.2 when comparing the distances $x$ and $y$ for the bead in the trap. The total energy of the molecular system is then given by $U(x, \lambda)=U_{m}(x)+(k / 2)(\lambda-x)^{2}$ where $U_{m}(x)$ is the energy of the molecular system. From Eq.(3) and using $\lambda=x+y$ we get $F=k y=k(\lambda-x)$. From Eq.(2) we then conclude that the mechanical work that satisfies the CFR is the accumulated work 
$W$ rather than the transferred work $W^{\prime}$. We remark a few relevant facts,

1. The transferred work $W^{\prime}$ does not satisfy the CFR and is dependent on the bandwidth of the measurement. The FDC and FEC are sensitive to the bandwidth or data acquisition rate of the measurement (Figure 6b). Whereas $W$ is insensitive to the bandwidth $W^{\prime}$ is not (see Figure 7a). This difference is very important because it implies that the bandwidth dependence implicit in the boundary term in Eq.(9) (the power spectrum of the force depends on the bandwidth if this is smaller than the corner frequency of the bead) is fully contained in $W^{\prime}$. Operationally it is much easier to use $W$ rather than $W^{\prime}$. As shown in Figure $7 \mathrm{~b}, W$ satisfies the CFR whereas $W^{\prime}$ does not. The logarithm of the ratio $\log \left(P_{F}\left(W^{\prime}\right) / P_{R}\left(-W^{\prime}\right)\right)$ plotted versus $W^{\prime} / k_{\mathrm{B}} T$ is strongly bandwidth dependent and exhibits a slope 30 times smaller than 1 (i.e. the slope expected for $W$ from the CFR) [27].

2. How big is the error committed in recovering free energy differences by using $W^{\prime}$ rather than $W$ ? Despite that $W$ and $W^{\prime}$ only differ by a boundary term (cf. Eq.9) one can show that, for the case of the mechanical folding/unfolding of the hairpin, the error in recovering free energy differences using the Jarzynski equality can be as large as $100 \%$ [27]. The error or discrepancy increases with the bandwidth. Interestingly enough, for small enough bandwidths (but always larger than the coexistence kinetic rates between the folded and unfolded states, otherwise the folding/unfolding transitions are smeared out) fluctuations in the boundary term in Eq.(9) are negligible and both $W$ and $W^{\prime}$ are equally good. This explains why previous experimental tests of the CFR that used $W^{\prime}$ instead of $W$ produced satisfactory results (e.g. [20]).

3. Inequivalence between moving the trap and the pipette or chamber. The noninvariance of the CFR under Galilean transformations suggests that moving the optical trap inside the fluidic chamber should not be necessarily equivalent to moving the pipette glued to the fluidic chamber. We have to distinguish two cases depending on whether the fluid inside the chamber is dragged (stick conditions) or not (slip conditions) by the moving chamber. The two scenarios are physically different because in the former case the bead in the trap is subject to an additional Stoke force due to the motion of the fluid. If the fluid is not dragged by the moving chamber (slip conditions) then $y$ is the right configurational variable. In this case, $U(y, \lambda)=U_{m}(\lambda-y)+(k / 2)(y)^{2}$ and the generalized force is equal to $F=U_{m}^{\prime}(\lambda-$ $y)$. Note that this $F$ is not equal to the instantaneous force measured by the optical trap but the instantaneous force acting on the molecule. Even in case of mechanical equilibrium the difference between the two instantaneous forces, $U_{m}^{\prime}(\lambda-y)$ and $k y$, produces a net non-negligible difference term. If the fluid does move with the chamber (stick conditions) then $x$ is again the right configurational variable and we recover the main results of this section. Interestingly, all experiments done until now that use motorized stages to move chambers operate in stick conditions so we do not expect experimental discrepancies for the definition of the work.

4. Other cases where the work definition matters. As we showed in Figure 7 the CFR and the right definition of work $W$ are both amenable to experimental test. Another interesting example where the boundary term is relevant is when the force $f$ (rather than the trap position) is controlled. As we saw in Section 1.1 the work 

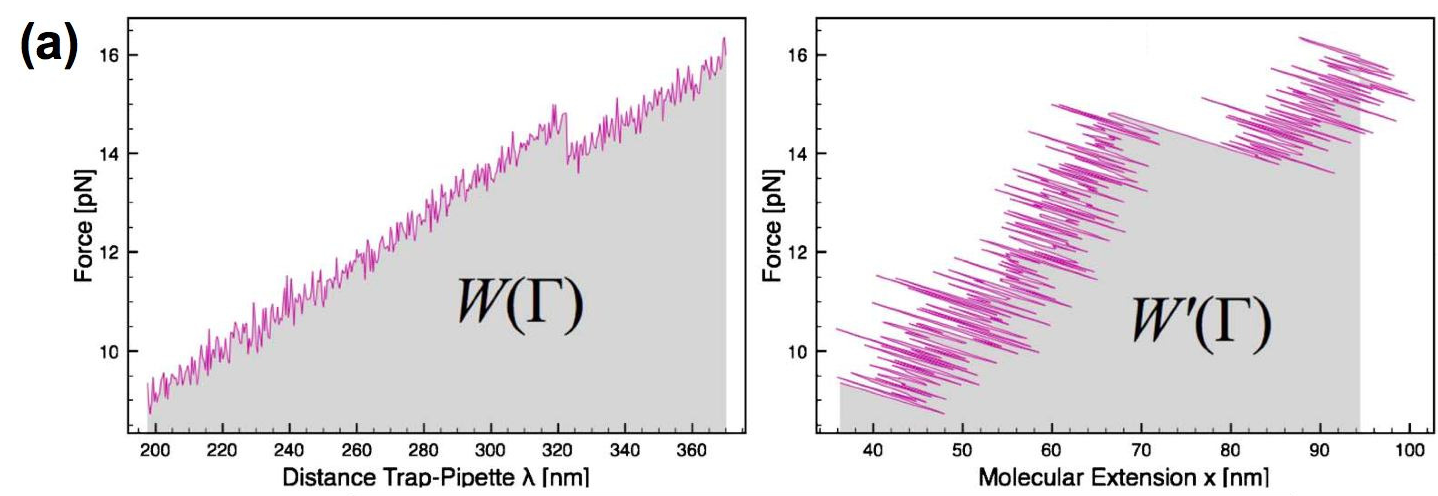

(b)

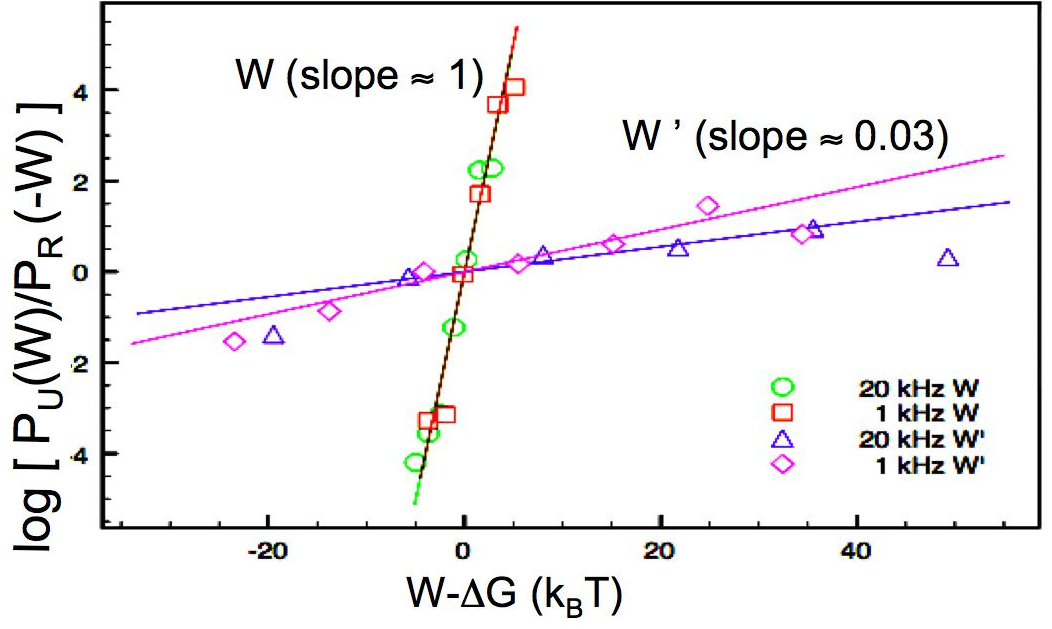

FIGURE 7. Accumulated $(W)$ versus transferred $\left(W^{\prime}\right)$ work. (a) The two work quantities for a given experimental trajectory. Note that the effect of bandwidth dependent force fluctuations is much larger for $W^{\prime}$ as compared to $W$, showing the importance of the boundary term Eq.9. (b) Experimental test of the CFR. When using $W$ the CFR is satisfied at all bandwidths. However when we use $W^{\prime}$ the CFR is strongly violated and dependent on the measurement bandwidth. Figure taken from [27].

in that case is given by $W_{X_{0}}=-\int_{f_{i}}^{f_{f}} X d f$ where $X=y+x+X_{0}$ is the absolute trap-pipette distance. Because $X_{0}$ stands for an arbitrary origin, the work $W_{X_{0}}$ is also a quantity that depends on $X_{0}$. This may seem unphysical but it is not [5]. The CFR is invariant respect to the value of $X_{0}$ as it can be easily checked by writing, $W_{X_{0}}=W_{X_{0}=0}-X_{0}\left(f_{f}-f_{i}\right)$, and using Eq.(8) gives $\Delta G_{X_{0}}=\Delta G_{X_{0}=0}-f X_{0}$. If the force $f$ is controlled, then other work related quantities such as $W^{\prime}=\int_{x_{i}}^{x_{f}} f d x$ or $W^{\prime \prime}=\int_{X_{i}}^{X_{f}} f d X$ differ from $W$ by finite boundary terms. Again these terms make the CFR not to be satisfied for $W^{\prime}$ and $W^{\prime \prime}$. These predictions are amenable to experimental test in magnetic tweezers (where the force is naturally controlled) or in optical tweezers operating in the force clamp mode with infinite bandwidth [28] (and possibly in a force feedback mode with finite bandwidth as well). 


\section{A GENERALIZED FLUCTUATION RELATION}

The CFR can be generalized to cases where the system is initially in partial, rather than global, equilibrium both in the forward and the reverse protocol [29]. Suppose we take a system at fixed control parameter $\lambda$ in thermal equilibrium with a bath at temperature $T$. The probability distribution over configurational variables $x$ is Gibbsian over the whole phase space $S$ meaning that: $P_{\lambda}^{\mathrm{eq}}(x)=\exp \left(-E_{\lambda}(x) / k_{\mathrm{B}} T\right) / Z_{\lambda}$ with $Z_{\lambda}$ the partition function $Z_{\lambda}=\sum_{x \in S} \exp \left(-E_{\lambda}(x) / k_{\mathrm{B}} T\right)$, where $E_{\lambda}(x)$ is the energy function of the system for a given pair $\lambda, x$. We refer to this condition as global thermodynamic equilibrium. However we might consider a case where the initial state is Gibbsian but restricted over a subset of configurations $S^{\prime} \subseteq S$. We refer to this case as partial thermodynamic equilibrium. Partially equilibrated states satisfy $P_{\lambda, S^{\prime}}^{\mathrm{eq}}(x)=P_{\lambda}^{\mathrm{eq}}(x) \chi_{S^{\prime}}(x) Z_{\lambda} / Z_{\lambda, S^{\prime}}$, where $\chi_{S^{\prime}}$ is the characteristic function defined over the subset $S^{\prime} \subseteq S\left[\chi_{S^{\prime}}=1\right.$ if $x \in S^{\prime}$ and zero otherwise], and $Z_{\lambda, S^{\prime}}$ is the partition function restricted to the subset $S^{\prime}$, i.e. $Z_{\lambda, S^{\prime}}=$ $\sum_{x \in S^{\prime}} \exp \left(-E_{\lambda}(x) / k_{\mathrm{B}} T\right)$. The partial free energy is then given by $G_{\lambda, S^{\prime}}=-k_{\mathrm{B}} T \log Z_{\lambda, S^{\prime}}$. Let us suppose again the scenario depicted in Figure 3. Along the forward process the system is initially in partial equilibrium in $S_{0}$ at $\lambda_{0}$. Along the reverse process the system is initially in partial equilibrium in $S_{1}$ at $\lambda_{1}$. The generalized CFR reads,

$$
\frac{p_{\mathrm{F}}^{S_{0} \rightarrow S_{1}}}{p_{\mathrm{R}}^{S_{0} \leftarrow S_{1}}} \frac{P^{S_{0} \rightarrow S_{1}}(W)}{P^{S_{0} \leftarrow S_{1}}(-W)}=\exp \left[\frac{W-\Delta G_{S_{0}, \lambda_{0}}^{S_{1}, \lambda_{1}}}{k_{\mathrm{B}} T}\right],
$$

where the direction of the arrow distinguishes forward from reverse, $p_{\mathrm{F}}^{S_{0} \rightarrow S_{1}}\left(p_{\mathrm{R}}^{S_{0} \leftarrow S_{1}}\right)$ stands for the probability to be in $S_{1}\left(S_{0}\right)$ at the end of the forward (reverse) process, and $\Delta G_{S_{0}, \lambda_{0}}^{S_{1}, \lambda_{1}}=G_{S_{1}}\left(\lambda_{1}\right)-G_{S_{0}}\left(\lambda_{0}\right)$ is the free energy difference between the partially equilibrated states $S_{0}$ and $S_{1}$.

Partially equilibrated states appear in many cases, from thermodynamic branches to intermediate and misfolded molecular states . The usefulness of the generalized CFR relies on our possibility to experimentally distinguish the substates visited along any trajectory and that these substates be visited frequently enough. For example, a molecule pulled by stretching forces can be in partial equilibrium when it stays either in the folded or unfolded state until it transits to the other state. If $S_{0}$ stands for the folded state and $S_{1}$ for the unfolded state, the generalized CFR makes possible to extract the free energies $G_{S^{\prime}}(\lambda)$ of the folded and unfolded states $S^{\prime}=S_{0}, S_{1}$ along the $\lambda$-axis, i.e. the folded and unfolded branches. Figure 8 shows an experimental verification of this result for a DNA haiprin that folds/unfolds in a two-states manner.

\section{CONCLUSION}

The possibility to experimentally measure the inverted motions remarked by Schrödinger more than half a century ago has boosted the study of energy fluctuations in very small objects under nonequilibrium conditions. The possibility to measure work fluctuations in single molecules that are mechanically unfolded has provided the testing ground for some of the most recent theoretical developments in nonequilibrium 


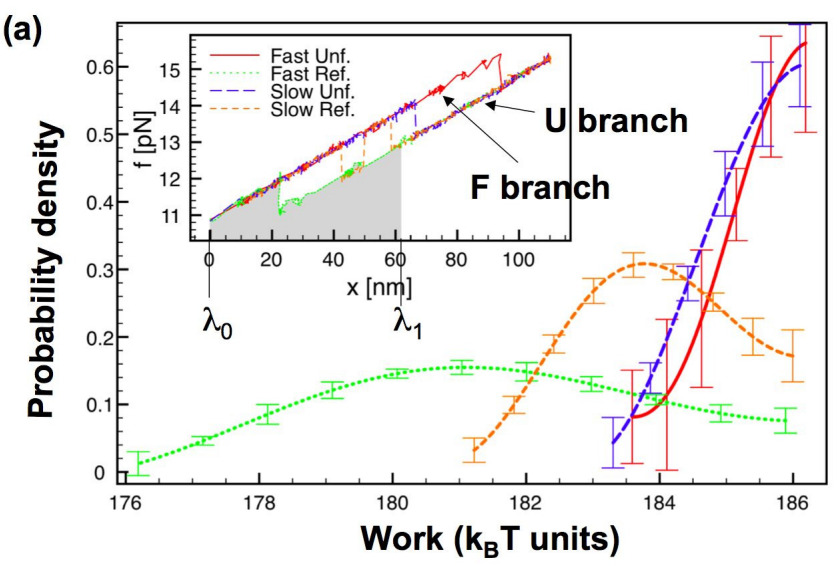

(b)

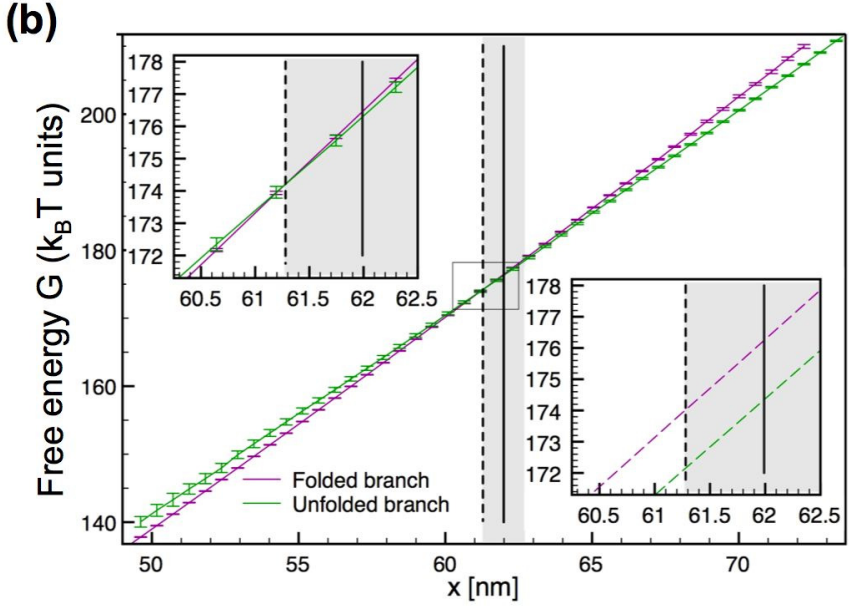

FIGURE 8. The generalized Crooks fluctuation relation. (a) Constrained work distributions measured in a $20 \mathrm{bp}$ hairpin at two different pulling speeds: $300 \mathrm{~nm} / \mathrm{s}$ (red, unfolding; green, refolding) and $40 \mathrm{~nm} / \mathrm{s}$ (blue, unfolding; orange, refolding). (a,Inset) The forward trajectories we consider are those where the hairpin starts partially equilibrated in the folded (F) state at $\lambda_{0}$ and ends in the unfolded (U) state (partially equilibrated or not) at $\lambda_{1}$. Note that, due to the correction term $p_{\mathrm{F}}^{F \rightarrow U} / p_{\mathrm{R}}^{F \leftarrow U}$ appearing in Eq.(10), restricted unfolding and refolding work distributions should not cross each other at a work value that is independent of the pulling speed. (b) Reconstruction of the folded (cyan color) and unfolded (green) free energy branches by applying the generalized CFR, Eq.(10), as shown in (a) and by varying the parameter $x \equiv \lambda_{1}$. The two branches cross each other around $x_{c} \simeq 82 \mathrm{~nm}$ corresponding to the coexistence transition. For $x<x_{c}\left(x>x_{c}\right)$ the $\mathrm{F}(\mathrm{U})$ state is the minimum free energy state. The upper left inset shows an enlarged view of the crossing region. The lower left inset shows the importance of the correction term $p_{\mathrm{F}}^{F \rightarrow U} / p_{\mathrm{R}}^{F \leftarrow U}$ appearing in Eq.(10). If that term was not included in Eq.(10) the coexistence transition disappears. Figure taken from [29].

statistical physics. Fluctuation relations and fluctuation theorems (e.g. the GallavottiCohen theorem for steady state systems [30]) are examples of new results that quantify inverted motions in nonequilibrium states. Measuring inverted motions has also practical applications: the Crooks fluctuation relation (CFR), Eq.(8), and its generalization to partial (rather than full) equilibrium conditions, Eq.(10), allows us to extract free energy differences between native or non-native states and free energies of thermodynamic branches. Future studies will also show the reliability of these methodologies to extract free energies of misfolded and intermediate states in RNAs or proteins, and base-pair free energies in nucleic acids unzipped under irreversible conditions. We also stressed how important is the correct definition of mechanical work to ensure the validity of the CFR. In this regard serious inconsistencies are encountered using other definitions of mechanical work but such inconsistencies are nowadays amenable to experimental test. Related to this, it is also important to underline the general non-invariance of fluctuation relations and theorems under Galilean transformations [31], an aspect that has not been stressed enough and that can also be tested in experiments. Finally, all the studies covered in this note address energy fluctuations of small classical systems under Gaussian noise conditions. It would be highly desiderable to have experiments done in systems in the regime of non-Gaussian noise (maybe at submicroseconds timescales), or in quantum systems where the concept of classical trajectory looses its usual meaning 


\section{ACKNOWLEDGMENTS}

A. A. and M. R. acknowledge financial support from the Spanish MEC-MICINN through the FPU fellowhip program, grant no. AP2007-00995 and Human Frontier Science Program (HFSP) (RGP55-2008) respectively. F. R acknowledges financial support from Grants FIS2007-3454. Human Frontier Science Program (HFSP) (RGP55-2008) and Icrea Academia prize 2008.

\section{REFERENCES}

1. Schrödinger E., What is life?, Cambridge University Press (1944).

2. Ritort F., J. Phys. Condens. Mat. 18, R531-R583.(2006).

3. Bustamante C., Liphardt J., Ritort F., Physics Today 5843 (2005)

4. Ritort F. Adv. Chem. Phys. 137, 31 (2008).

5. Peliti L., J. Stat. Mech. P05002 (2008) and references therein

6. Wang G. M., Sevick E. M., Mittag E., Searles D. J., and Evans D. J., Phys. Rev. Lett. 89, 050601 (2002)

7. Mazonka O. and Jarzynski C., e-print cond-mat/991212

8. Van Zon R. and Cohen E. G. D., Phys. Rev. Lett. 91, 110601 (2003)

9. Garnier S. and Ciliberto S., Phys. Rev. E, 71 060101R (2005)

10. Evans D. J. and Searles D. J., Phys. Rev. E 501645 (1994)

11. Crooks G. E., Phys. Rev. E 602721 (1999)

12. Jarzynski C., Phys. Rev. Lett. 78, 2690 (1997)

13. Hummer G. and Szabo, Proc. Nat. Acad. Sci. (USA) 98, 3658 (2001)

14. Liphardt J., Dumont S., Smith S. B., Tinoco Jr. I. and Bustamante C., Science 296, 1832 (2002)

15. Zuckerman D. and Woolf T., Chem. Phys. Lett., 351, 445 (2002); Phys. Rev. Lett. 89, 180602 (2002).

16. Ritort F., Bustamante C. and Tinoco Jr. I., Proc. Nat. Acad. Sci. (USA) 99, 13544 (2002)

17. Bennett C. H., J. Comp. Phys. 22, 245 (1976)

18. Shirts M. R., Bair E., Hooker G. and Pande V. S., Phys. Rev. Lett. 91, 140201 (2003)

19. Minh D. D. L. and Adib A. B., Phys. Rev. Lett. 100, 180602 (2008)

20. Collin D., Ritort F., Jarzynski C., Smith S. B., Tinoco Jr. I. and Bustamante C., Nature 437, 231 (2005)

21. Mossa A., Manosas M., Forns N., Huguet J. M. and Ritort F., J. Stat. Mech. P02060 (2009)

22. Manosas M., Mossa A., Forns N., Huguet J. M. and Ritort F., J. Stat. Mech. P02061 (2009)

23. Huguet J. M., Bizarro C. V., Forns N., Smith S. B., Bustamante C. and Ritort F., Proc. Nat. Acad. Sci. (USA) 107, 15431 (2010)

24. Woodside M T, Behnke-Parks W M, Larizadeh K, Travers K, Herschlag D and Block S M, Proc. Nat. Acad. Sci. 103, 6190 (2006)

25. Forns N., De Lorenzo S., Manosas M., Huguet J. M. and Ritort F., submitted for publication

26. Schurr J. M. and Fujimoto B. S., J. Phys. Chem. B 107, 14007 (2003)

27. Mossa A., De Lorenzo S., Huguet J. M. and Ritort F., J. Chem. Phys. 130, 234116 (2009)

28. Greenleaf W. J., Woodside M. T., Abbondanzieri E. A. and Block S. M., Phys. Rev. Lett. 95, 208102 (2005).

29. Junier I., Mossa A., Manosas M. and Ritort F., Phys. Rev. Lett. 102, 070602 (2009)

30. Gallavotti G. and Cohen E. G. D., Phys. Rev. Lett. 742694 (1995)

31. Speck T., Mehl J., and Seifert U., Phys. Rev. Lett. 100, 178302 (2008)

32. Ritort F., Physics 2, 43 (2009) 